MAGNETOHYDRODYNAMICS Vol. 54 (2018), No. 1-2, pp. 121-125

DOI: $10.22364 / \mathrm{mhd} .54 .1-2.21$

\title{
MEASUREMENT OF THE VAPOR BUBBLE FORMATION FREQUENCY IN BOILING MAGNETIC FLUID BY A TWO-LAYER MEDIUM METHOD
}

\author{
A. Yanovskii ${ }^{1}$, A. Simonovskii ${ }^{1,2}$, I. Chuenkova ${ }^{2}$ \\ 1 Department of Physics, Stavropol State Agrarian University, \\ 12 Zootekhnicheskiy per., 355017 Stavropol, Russia \\ ${ }^{2}$ Department of General and Theoretical Physics, North-Caucasus Federal University, \\ 2 Kulakov ave., 355029 Stavropol, Russia \\ e-Mail: aa.yanovskiy@yandex.ru
}

Boiling of a magnetic fluid is of interest in the hardening steel technology for nondeformation cooling with a given distribution of the current and stresses. The character of the heat transfer during boiling essentially depends on the formation, growth and detachment of the vapor bubbles. In this paper, we investigate mechanisms of the magnetic field influence on the kinetic parameters of the bubble boiling process in a magnetic fluid, in particular, on the formation frequency of vapor bubbles.

1. Experiment. In the experiments, the heating surface was heated by a point source and, as a consequence, only one vaporization center was operated during the boiling of magnetic fluid (MF).

The optical methods are the most effective for the observation of bubbles formation dynamics at fluids boiling. However, magnetic fluids are opaque, which makes it impossible to use these methods in practice. Also known is a system of induction coils for measuring the frequency formation of vapor bubbles [1]. But using this method, a vapor bubble should arise and grow asymmetrically to the geometrical axis of the system which is not always achievable.

In the present work, the formation frequency of vapor bubbles during the boiling of a magnetic fluid was measured by a two-layer medium method: a layer of water was poured over the magnetic fluid surface. This allowed to visualize the processes of formation and emergence of vapour bubbles during the transition of the interface between a transparent and an opaque medium, and also to characterize the formation frequency of the vapor bubbles by time-lapse analysis of the boiling process records.

A device for the measurement of the vapor bubble formation frequency in a boiling magnetic fluid on an unrestricted surface is shown in Fig. 1. A magnetic fluid (1) was poured into a cylindrical quartz glass vessel (2), mounted on a heating steel plate (3) and boiled there. A water layer of the same height was poured over the layer of the magnetic fluid (4). The water density is less than that of the kerosene-based MF we used, therefore, the poured water was above the magnetic fluid, and both layers did not mix. Heat was supplied to the central part of the heating plate by a rod (5) $4 \mathrm{~mm}$ in diameter. Thus, only one vapor center was operated during the boiling process. The heating plate was made of a non-magnetic material (austenitic steel with the magnetic permeability $\mu<1.05$ ). The conjunct two chromel-cope-like thermocouples (6) positioned $5 \mathrm{~mm}$ apart registered the heating surface temperature and controlled the heat flux coming into the fluid along the rod. The temperature of the heating surface was sampled by a top thermocouple (3). The readings of the top and bottom thermocouples made it possible to calculate the heat flux into the fluid. An electric heater $(7)$ with a 


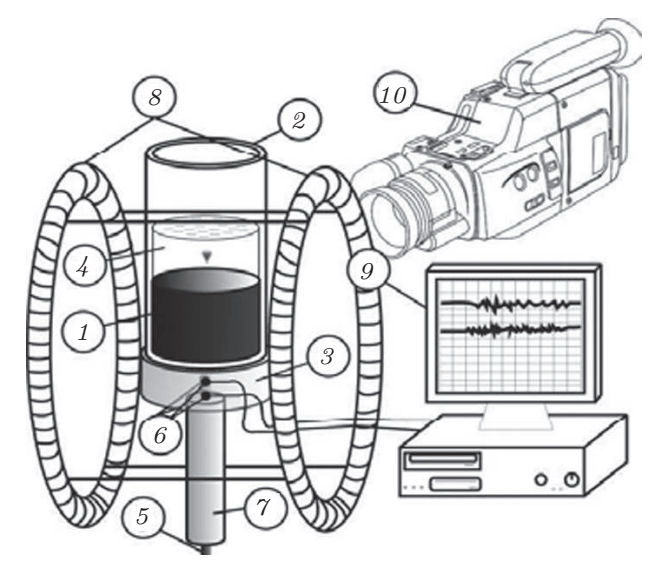

Fig. 1. The experimental device.

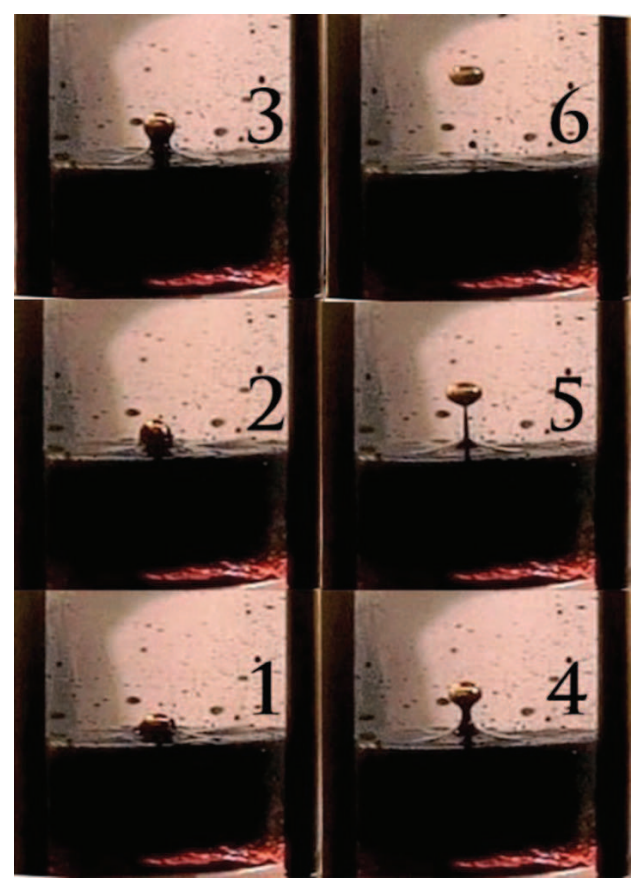

Fig. 2. Emergence of a vapor bubble in a layer of water.

bifilar winding was installed on the rod (5) to compensate the internal magnetic field of the heater winding.

The vessel with the MF was exposed to the uniform magnetic field of Helmholtz coils (8), so that the vaporizing center on the heat transfer surface coincided with the geometric center of the coils. The vector of the magnetic field of the coils could be directed parallel (horizontal magnetic field) or perpendicular (vertical magnetic field) to the plane of the heating surface. The signals from the thermocouple were recorded by a computer (9) equipped with an analog-to-digital converter.

With simultaneous supply of voltage to the heating steel plate and to the Helmholtz coils, the thermocouple signals were recorded. 
Measurement of the vapor bubble formation frequency in boiling magnetic fluid...

The boiling process was recorded by a video camera (10) at a rate of 600 frames per second. The number of bubbles appearing on the surface of the magnetic fluid per unit time was detected by time-lapse counting.

The images displayed in Fig. 2 were taken at the frame-by-frame decomposition of a high-rate review and demonstrate the occurrence of a vapor bubble in a layer of water. Separate frames 1-6 in Fig. 2 demonstrate video shots taken at successive time moments, starting with the moment when the bubble passes through the magnetic-liquid interface of the water vapor. In each frame, the glass vessel in the lower part is filled with a black opaque magnetic fluid and the clear water is at the top of the vessel. A mushroom-shaped vapor bubble at the center of the picture passes through the interface-transparent water.

\section{Results and discussion.}

2.1. Horizontal uniform steady magnetic field. Fig. 3 shows the dependence of the formation frequency of vapor bubbles on the temperature of the heating surface at different intensities of the magnetic field.

The dependences illustrated in this figure were obtained for a kerosene-based magnetic fluid. The vapor bubbles begin to float at the interface at the temperature of the heating surface $t=112-120^{\circ} \mathrm{C}$. It is also seen from the graph that the vapor bubble formation frequency increases monotonically with the temperature of the heating surface over the entire temperature range of the heater. Curve 1 characterizes the change in vapor bubble formation frequency with increasing temperature of the heating surface in the zero magnetic field. Curve 2 describes the same process under the external horizontal uniform magnetic field $0.7 \mathrm{kA} / \mathrm{m}$, etc.

The statistical analysis of the experimental data has shown that in the temperature range of the heating surface $112-125^{\circ} \mathrm{C}$, the difference for curves $1-7$ is not statistically significant, which indicates a weak influence or absence of the influence of the magnetic field on the vapor bubble formation frequency in this temperature range. However, at temperatures of the heating surface above $130^{\circ} \mathrm{C}$, a $15 \%$ difference for curves 1-7 becomes statistically significant. At a heating surface temperature of $145^{\circ} \mathrm{C}$, the vapor bubble formation frequency presented by curves $1-7$ decreases with increasing magnetic field strength by more than $30 \%$.

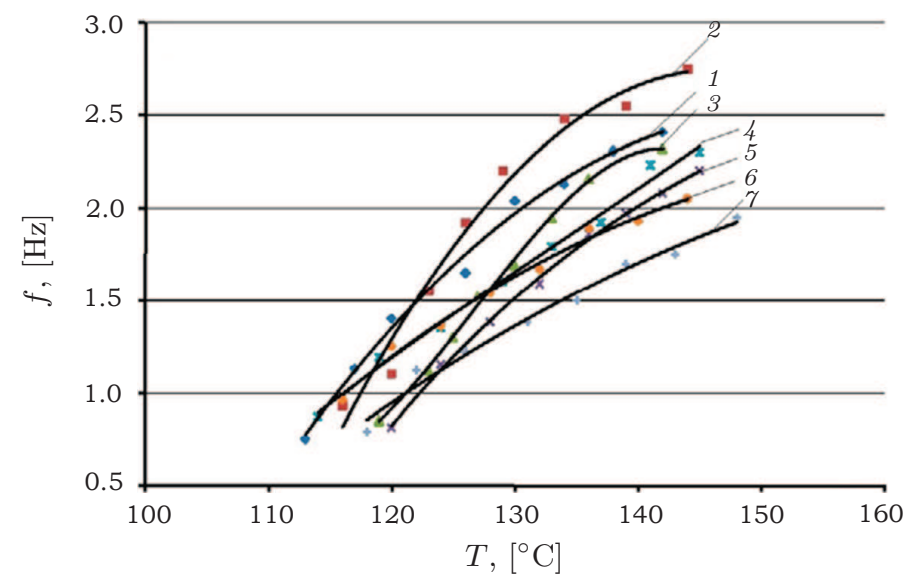

Fig. 3. The dependence of the vapor bubbles formation frequency on the temperature of the heating surface at different intensities of the horizontal magnetic field, $\mathrm{kA} / \mathrm{m}: 1-$ $0,2-0.7,3-1.4,4-2.1,5-2.8,6-3.5,7$ to 4.2 . 


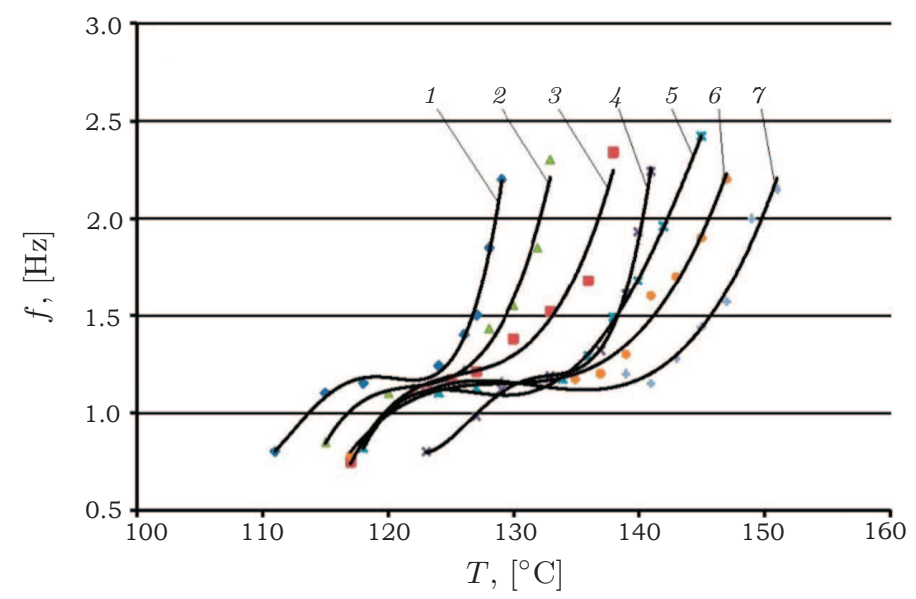

Fig. 4. The dependence of the vapor bubble formation frequency on the temperature of the heating surface at different intensities of the vertical magnetic field, $\mathrm{kA} / \mathrm{m}: 1-0$, $2-0.7,3-1.4,4-2.1,5-2.8,6-3.5,7$ to 4.2 .

2.2. Vertical uniform steady magnetic field. Fig. 4 shows the dependences obtained when the MF sample boils in a vertical magnetic field. As seen from the graph, the beginning of recording the boiling process starts in the temperature range of the heating surface $110-123^{\circ} \mathrm{C}$.

Curves 1-7 increase monotonically with increasing temperature of the heating surface and are similar to each other. However, the curves obtained in stronger magnetic fields are shifted along the abscissa axis toward higher temperatures relative to the curves obtained in weak magnetic fields or without the field.

Similar measurements in a horizontal magnetic field turned out to be impossible because of the peculiarities of our method. The layers of magnetic fluid and water were mixed, producing an emulsion. It was not possible to fix a vapor bubble and to record video.

3. Conclusions. The experimentally observed effect of the magnetic field on the formation, growth and detachment of vapor bubbles at magnetic fluid boiling can be elucidated by many factors. The most significant of them are the magnetic field acting on the vapor bubble formation in a boiling magnetic fluid and the mechanical forces acting on the vapor bubble on the heated surface and forming a mechanical equilibrium of the surface heating, similar to the boiling of ordinary liquids. Among these mechanical forces we can identify the adhesion of the vapor bubble by the heating surface determined by the edge angle and its dependence on the magnetic field, the Archimedes force resulting from the gradient of hydrostatic pressure, as well as possible magnetic forces influencing the mechanical equilibrium of a vapor bubble on the heating surface. These magnetic forces can be caused by the temperature gradient in the magnetic fluid near the heater surface in a thermal boundary layer. The temperature gradient in the thermal boundary layer leads to the formation of the gradient magnetic field at the heater surface of the magnetic fluid, the magnetization fluid gradient and the magnetic susceptibility gradient of the fluid. All listed above forces and moments acting on the vapor bubble are partially presented and differently interpreted in $[1,2]$.

Acknowledgements. The work was carried out with financial support of the Russian Foundation for Basic Research (Grant No. 17-01-00037). 
Measurement of the vapor bubble formation frequency in boiling magnetic fluid...

\section{References}

[1] M.A. Kobozev, A.YA. Simonovskil. Formation rate of vapor bubbles in magnetic fluid boiling at a single vaporization center: measuring technique and experimental setup. Technical Physics, vol. 52 (2007), no. 11, pp. 14221428 .

[2] A.A. Yanovskiy, A.Ya. Simonovsky, V.L. Kholopov, I.Yu. ChuenKOVA. Heat transfer in boiling magnetic fluid in a magnetic field. Solid State Phenomena, vol. 233-234 (2015), pp. 339-343.

Received 27.12.2017 